\title{
DEPENDENCIES OF BREAKING TYPE, BREAKING CRITERIA AND ENERGY DISSIPATION ON AMPLITUDE-PHASE FREQUENCY STRUCTURE OF WAVES
}

\author{
Sergey Kuznetsov ${ }^{1}$, Yana Saprykina ${ }^{2}$ and Valentina Volkova ${ }^{3}$
}

\begin{abstract}
Type of wave breaking - plunging or spilling - depends on symmetry of waves. The spilling waves are asymmetric against horizontal axis and are practically symmetric against vertical axis so the phase shift between first and second nonlinear harmonics (or biphase) is close to zero. The plunging breaking waves have larger asymmetry against vertical axis, (biphase is close to $-\pi / 2$ ), and near symmetric on horizontal axis (close to saw-toothed form). Non-linear wave transformation influences on depth-induced wave breaking. Breaking index depends on relation of wave energy in frequency range of second nonlinear harmonics to wave energy in frequency range of main harmonic and on biphase. The dissipation rate of spilling breaking waves energy quadratically depends on frequency, while in plunging breaking, this dependency is practically linear for all frequencies.
\end{abstract}

Keywords: wave energy dissipation; wave breaking; spilling; plunging; amplitude phase frequency structure

\section{INTRODUCTION}

Many features of processes of wave energy dissipation in the surf zone, important for sediments transport and changing of bottom relief, are remain studied non-sufficient. For example, the type of wave breaking are predicted on the base of empirical not physically grounded parameters, the depthinduced wave breaking criteria widely used in different models based on linear dependence between height of breaking wave $(H)$ and depth of water in breaking point $(h): \gamma=h / H$ that not confirmed by the experiments. With adjustable constant or breaking index $\gamma=0.8$ (for heights of individual waves (Battjes and Janssen 1978) or $\gamma=0.6$ (for significant wave height (Thornton and Guza 1983)) is suitable for most wave breaking cases, but many experimental data, including our, testify the wide range of $\gamma$ : from 0.4 up to 1.2 (Saprykina et al. 2017). To explain its variations the dependence on bottom slope and wave steepness was assumed, but this dependence only as a tendency is observed (Battjes 1974). We suppose that the variations of $\gamma$ and type of wave breaking can be explained by the distinctions of the amplitudephase frequency (spectral) structure of waves before breaking due to nonlinear wave transformation. The frequency selectivity of wave energy dissipation depends on nonlinear process of wave transformation and organized in the manner to compensate the results of it (Kuznetsov and Saprykina 2004). The main goal of this work is to testify the influence the nonlinear structure of coastal zone waves on their breaking and energy dissipation features.

\section{EXPERIMENTS}

We will use the results of two field and three laboratory experiments. In advance we tell that all of our experiments give the identical results concerning the considered problems.

\section{Field conditions}

We analyze the results of two field experiments "Shkorpilovtsy -2007" and "Shkorpilovtsy -2016", produced at the same polygon of Institute of oceanology of Bulgarian Academy of science at Bulgaria, Black Sea, were 15 or 22 wire wave gauges was installed from the trestle over the barred sandy bottom of mean slope 0.025 , that register the wave chronograms synchronously at sampling rate $20 \mathrm{~Hz}$ during the month. The view on the trestle, bottom profiles and gauge positions are shown at Fig. 1. The place and type of wave breaking was fixed visually and by video and photo recoding.

\section{Laboratory conditions}

Two of three laboratory experiments was produced at Research center "Sea Shores" Sochi, Russia, in 2004 and in 2013, 8 or 15 capacitance type wave gauges was installed over the bottom slopes of 0.04 and 0.022 respectively. The last experiment was produced at Tainan Hydraulics Laboratory,

\footnotetext{
${ }^{1}$ Zenkovich Coast and Shelf Laboratory, Shirshov Institute of Oceanology RAS, Nakhimovskii pr., 36, Moscow, 117997, Russian Federation

${ }^{2}$ Zenkovich Coast and Shelf Laboratory, Shirshov Institute of Oceanology RAS, Nakhimovskii pr., 36, Moscow, 117997, Russian Federation

${ }^{3}$ Geography Faculty, Lomonosov Moscow State University,GSP-1, Leninskie Gory, Moscow, 119991, Russian Federation
} 


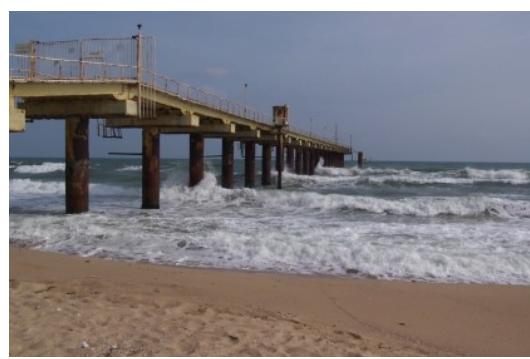

a)

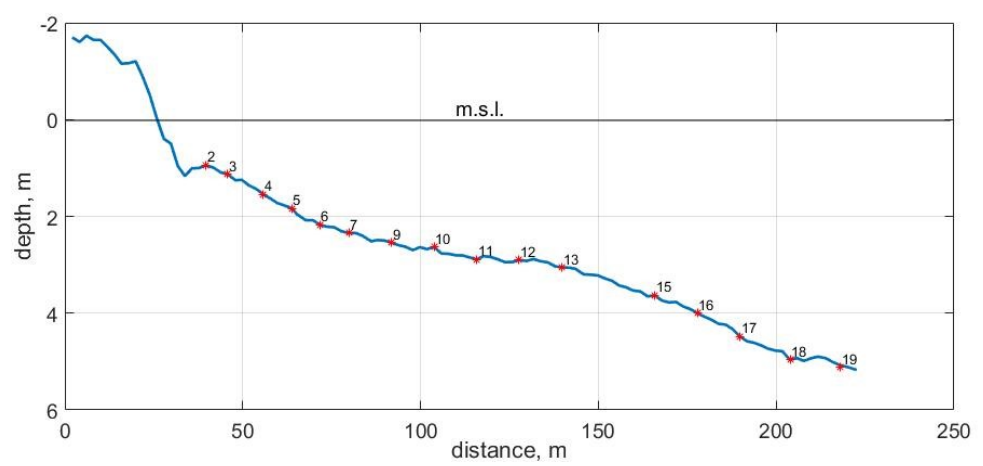

c)

Figure 1. Bottom profiles and gauge positions in field experiment "Shkorpilovtsy -2007" (b), in "Shkorpilovtsy -2016"(c) and view on the trestle (a). Shkorpilovtsy, Bulgaria, Black sea coast.
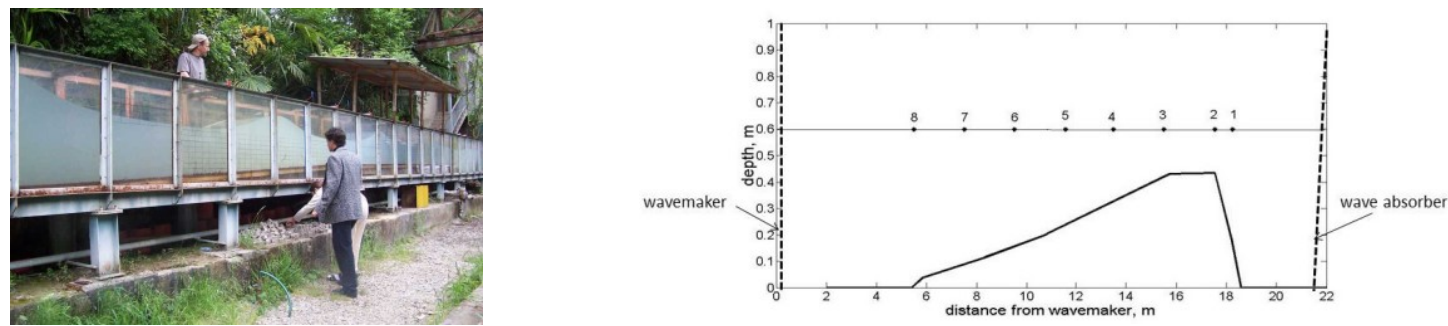

a)
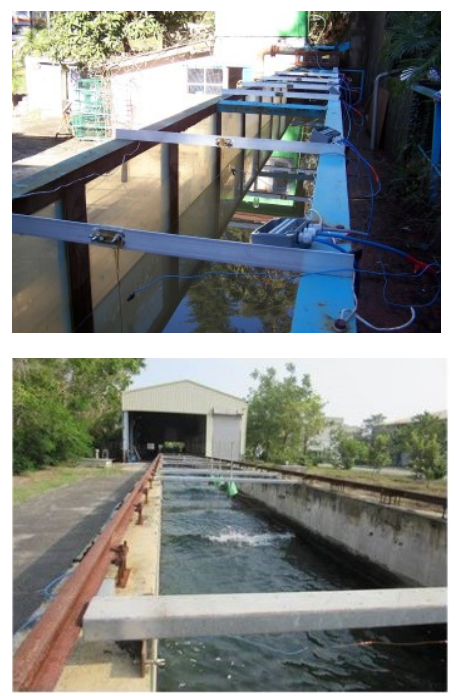

b)
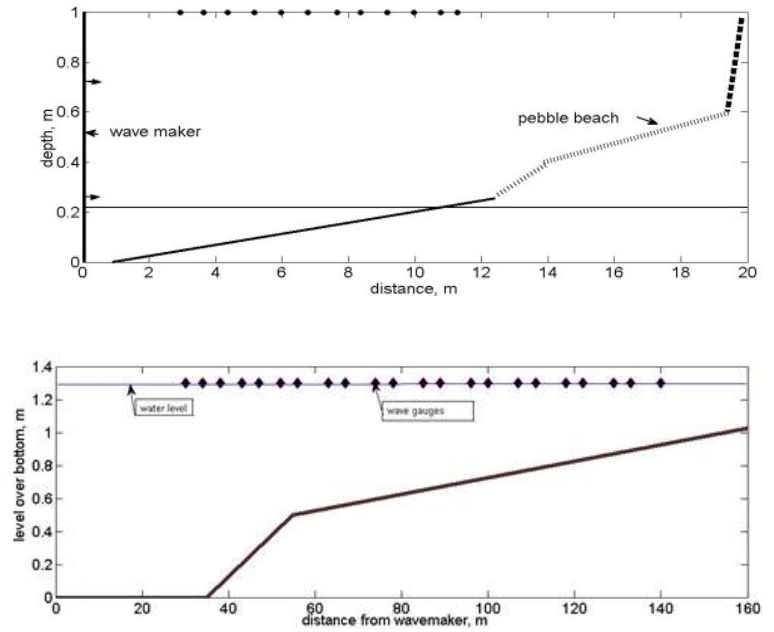

c)

Figure 2. The views on experimental flumes and bottom profiles and gauge positions in three laboratory experiments. (a) - 2004, (b) - 2013, (c) - 2015

Taiwan, 2015, where 15 capacity type wave gauges over bottom slope 0.005 . The initially monochromatic wave was used in all of the experiments. The flumes and bottom profiles are shown at Fig. 3 and 4. The wave gauges records the wave chronograms synchronously at sampling rate $20 \mathrm{~Hz}$ during the 3-6 minutes for each series of measurements. The place and type of wave breaking was fixed 
visually and by video and photo recoding. The views on experimental flumes, bottom profiles and wave gauge positions are shown at Fig. 2.

\section{SYMMETRY OF BREAKING WAVES}

For coastal zone wave transformation process the space periodical energy exchange between the first and second harmonics is typical (Saprykina et al. 2013). We will consider this scenario of wave transformation in field and laboratory conditions to look more in details the difference of structure of waves breaking by spilling and plunging type. At such case two lines of wave breaking exist in the coastal zone. The first line is the spilling breakers and the last one - plunging breaking. The different type of breaking provided by different amplitude-frequency content of waves in lines of breaking and by different phase shifts (biphase) between first and second harmonics of waves. Typical spectra evolutions for laboratory and field condition are shown at Fig. 3. In field conditions (Fig. 3b) the waves propagate from distance $240 \mathrm{~m}$ to the shore at distance $40 \mathrm{~m}$. The energy of first harmonic (frequencies about $0.15 \mathrm{~Hz}$ ) decrease to the minimum at $150 \mathrm{~m}$ and then grove again. The second harmonic amplitude (frequencies about $0.3 \mathrm{~Hz}$ ) evaluate in antiphase to the first one as shown at Fig. $4 \mathrm{~b}$, the place and type of breaking marked on the figures.

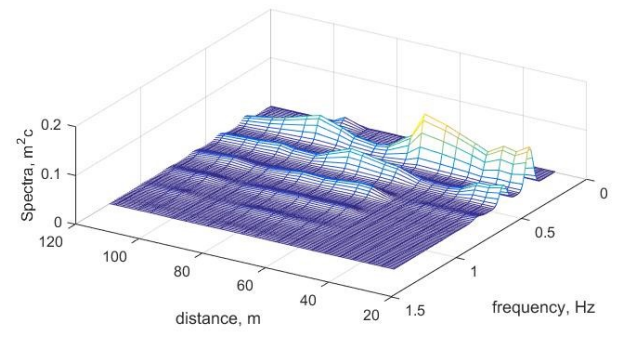

a)

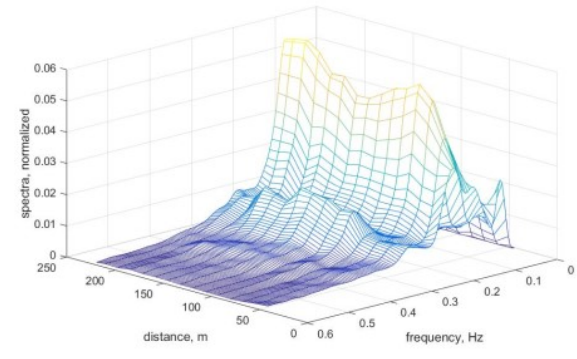

b)

Figure 3. Spectral evolution of waves in laboratory (a) and field (b) conditions.

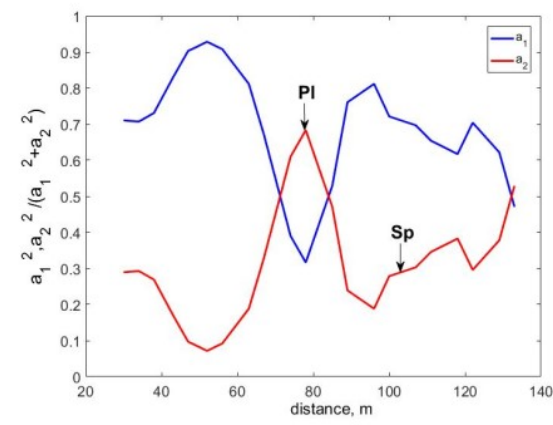

a)

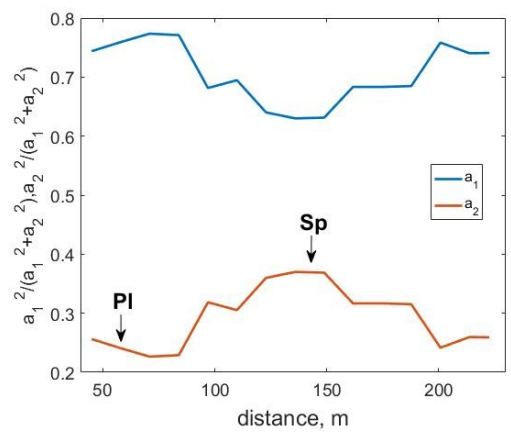

b)

Figure 4. Spatial evolution of first and second harmonic amplitudes in laboratory (a) and field (b) conditions. Amplitudes calculating by integratiog of spectra in proper range.

From Fig. 3 and 4 we can conclude that plunging breaking waves have the relative small second harmonic amplitude than spilling one.

The sample of spilling and plunging wave chronograms and their wavelet transform are shown on Fig 5 and 6 for laboratory and field conditions and demonstrate that crests of waves of second harmonics are shifted forward against the crests of first ones in direction of propagation. In spilling waves the positions of crests of waves of second harmonics coincide with the positions of the crests of first ones. 

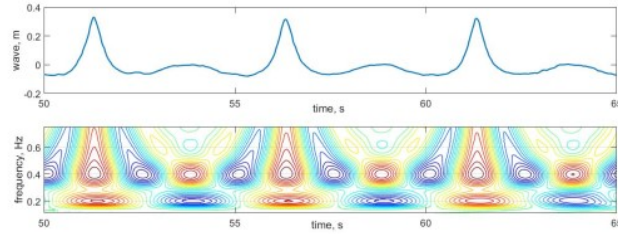

a)

Figure 5. Sp (a) and PI (b) breaking waves chronograms at the laboratory conditions and its wavelet transforms.
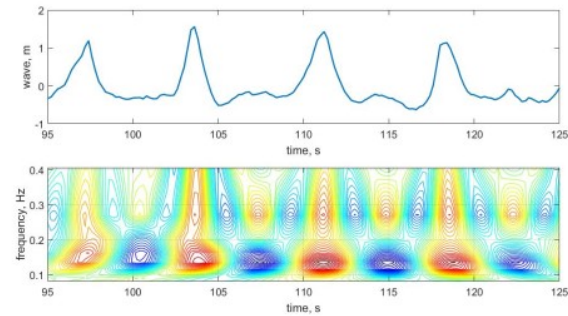

a)

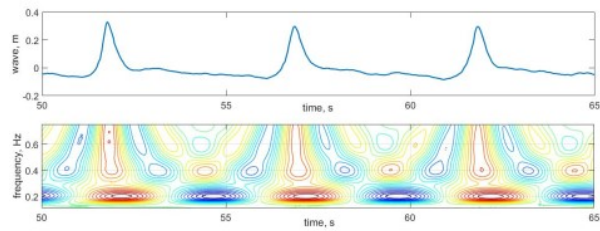

b)
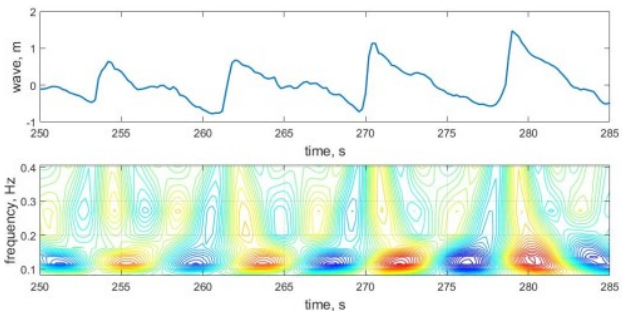

b)

Figure 6. Sp (a) and PI (b) breaking field conditions waves and its wavelet transforms.

From Fig. 3-6 we can conclude that plunging breaking waves - "saw-tooth" type waves with steep forward front, symmetrical on horizontal axis, second nonlinear harmonics amplitude is relatively small. Spilling breaking waves - waves with sharp and high crest and flat trough, symmetrical on vertical axis, second nonlinear harmonics amplitude is relatively high. Periodical in space energy exchange of energy between the first and second harmonics is the reason the phenomena that same waves breaking on the same mean slope by different types that contradict to the empirical criteria described the type of wave breaking as dependence on Iribarren (or on similar) number.

\section{“NONLINEAR" DEPENDENCIES OF WAVE BREAKING POSITION}

Spectral composition of waves effects on breaking index $\gamma$. It depends on relative size of energy of second nonlinear harmonic of waves at breaking point (Saprykina et al. 2017). An increase of $\gamma$ is characteristic of waves in which the amplitude of the second harmonic $\left(E_{2} / E_{1}\right)$ is approximately less that $0.35: \gamma=H s / h_{b}=0.6+3.5\left(E_{2} / E_{1}\right)^{2}$. For waves with $\left(E_{2} / E_{1}\right)>0.35 \%, \gamma$ does not increase and has approximately uniform distribution with respect to its mean value of 0.6 as shown at Fig. 5 .

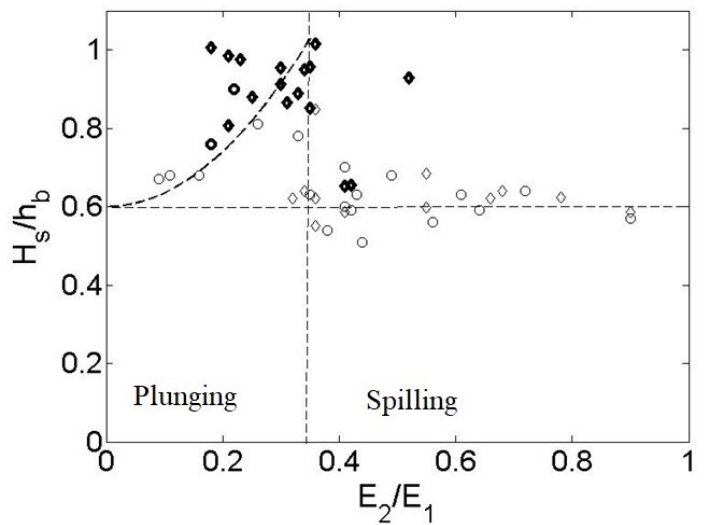

Figure 7. The dependence of breaking index on relative value of second harmonic. Circles - a laboratory experiment, diamonds - field data, stars - numerical results. Bold symbols correspond to the waves breaking by plunging, while the usual ones correspond to the waves breaking by spilling. Modified from Saprykina et al. 2017. 
Dependence of relative energy of second harmonic on Ursell number and wave steepness at breaking point was determined empirically also (Fig. 7). For small Ursell numbers $(\mathrm{Ur}<1)$, the relative energy of the second nonlinear harmonic in the experimental data corresponds well to weakly nonlinear dispersive Stokes waves and can be determined by formula

$$
\frac{a_{2}}{a_{1}} \approx \frac{3}{4} \frac{a k}{(k h)^{3}}=\frac{3}{4} \mathrm{Ur}
$$

These waves break predominantly by spilling.

With an increase in the Ursell number and, accordingly, an increase in the influence of nonlinearity, the relative share of energy of the second harmonic is not described by relation. Such waves break predominantly by plunging.

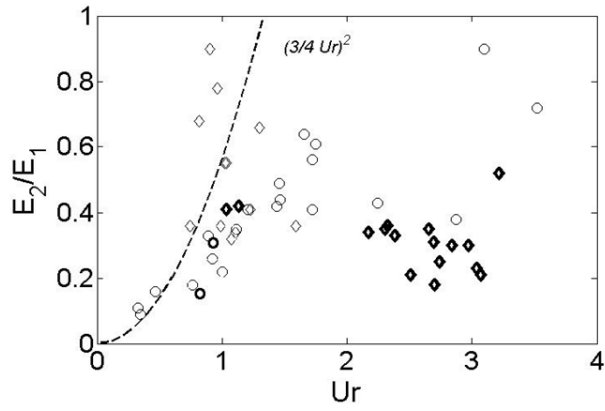

a)

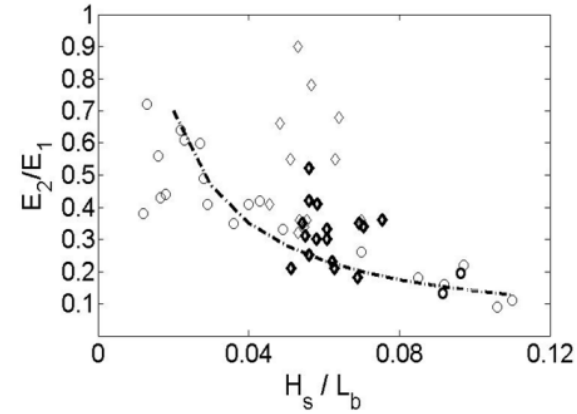

b)

Figure 8. The dependencies that permit us to determine the relative amplitude of second harmonic of waves by Ursell number (a) and by wave steepness at breaking point (b). The keys are as on Fig. 7. Modified from Saprykina et al. 2017.

\section{FREQUENCY DEPENDENCIES OF WAVE ENERGY DISSIPATION ON BREAKING TYPE}

The dynamic of wave transformation at the breaking point is affected both by dissipation and nonlinear processes. In order to separate the input of dissipation and non-linear transformation, the experimental data was imposed to numerical modeling with the use of Boussinesq-type equations with improved dispersion characteristics (Madsen and Sorensen 1993; Eldeberky and Battjes 1996), follow the method of (Kuznetsov and Saprykina 2004). The equations were solved in spectral domain, and altogether they form a set of evolution equations for non-linear complex Fourier amplitudes $\left(A_{p}\right)$ :

$$
\frac{d A_{p}}{d x}=-\beta_{2} \frac{h_{x}}{h} A_{p}-i 2 g\left(F_{p}^{+}+F_{p}^{-}\right)-\alpha_{n} A_{p}
$$

The first term of the right side of (2) describes the linear increase of amplitude, when wave approaches the shallow water zone, and this term is proportional to slope steepness $\left(h_{x}\right)$ and wave amplitude at the particular point. The second and third terms represent, respectively, the summary and incremental triad interaction of harmonics, described by:

$$
\begin{aligned}
& F_{p}^{-}=\sum_{m=1}^{\infty} \frac{\alpha^{-}}{\beta_{1}} A_{m}^{*} A_{p+m} \exp \left[-i\left(\psi_{m+p}+\psi_{m}+\psi_{p}\right)\right], \\
& F_{p}^{+}=\sum_{m=1}^{p-1} \frac{\alpha^{+}}{2 \beta_{1}} A_{m} A_{p-m} \exp \left[-i\left(\psi_{m}+\psi_{p-m}+\psi_{p}\right)\right],
\end{aligned}
$$

where $g$ is the gravitational force, $\beta_{l}, \alpha^{+}$and $\alpha^{-}$are interaction coefficients, that depend on local depth $(h)$, wave number $\left(k_{p}\right)$, and frequency $\left(\omega_{p}\right) ; \alpha_{n}$ is the dissipation rate, which is usually derived empirically, $\psi_{\mathrm{p}}$ is the phase, derived from

$$
\frac{d \psi_{p}}{d x}=k_{p},
$$

where $\omega_{p}$ is angular frequency, $k_{p}$ is the wave number.

The dissipation rate assessment method, based on comparing modeled (dissipation is negliged) and experimental (measurements, naturally including the effects of non-linear transformation and dissipation) of wave spectra, was suggested and laid out in (Mase , Kirby, 1992). The dissipation rate of waves propagating towards coast may be estimated with this formula:

$$
\alpha_{n}=\frac{\left(S\left(x_{\text {start }}+\Delta x\right)_{\text {calc }}-S\left(x_{\text {start }}+\Delta x\right)_{\text {meas }}\right)}{S\left(x_{\text {start }}\right)_{\text {meas }} \times(2 \Delta x)},
$$


where $S$ is wave spectra at specified location, calc and meas indices apply to modeled and measured spectra, respectively, $\Delta x$ is the distance crossed by waves, $x_{\text {start }}$ is the coordinate of the starting point, where calculations begin.

Fig.9 represents the dissipation rate, calculated with the use of (5) for monochromatic waves during different breaking events. It can be observed, that in case with spilling breaking, the dissipation rate quadratically depends on wave frequency, while in plunging breaking, this dependency is virtually linear and almost constant for all frequencies.

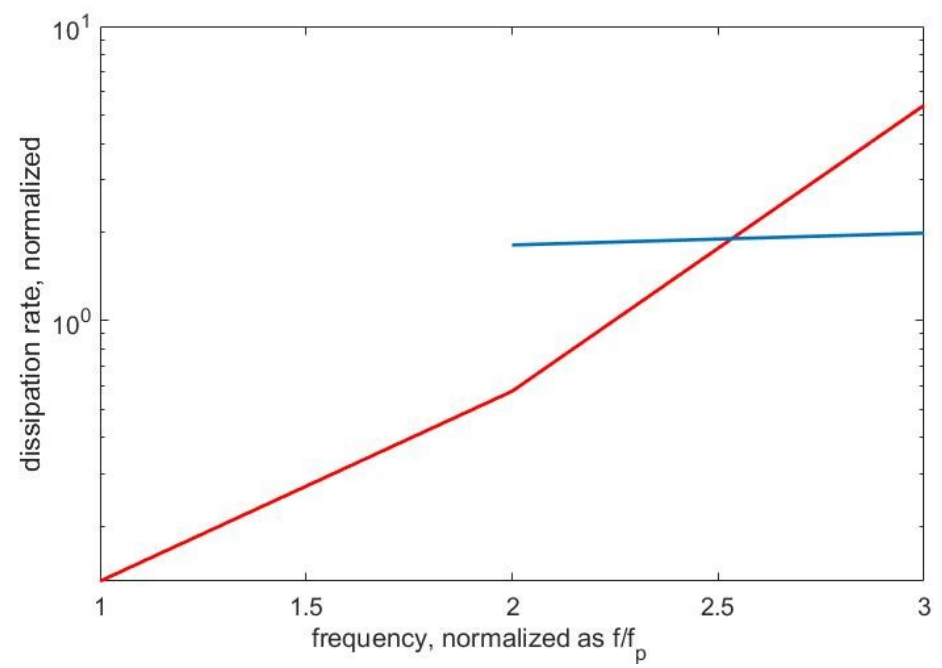

Figure 9: The dimensionless dissipation rate, calculated by using of (5) in case of laboratory experiments. Red line stands for spilling breaker, blue line stands for plunging breaker.

That way, the dissipation rate, related with the energy decrease during breaking, significantly differs, depending on the type of breaking event. The dissipation rate, which is equal to a constant, was suggested in (Eldeberky and Battjes 1996), and corresponds to plunging breaking, while for spilling breaking it requires quadratic parametrization (Mase and Kirby 1992).

\section{CONCLUSIONS}

As we seen from the laboratory and field experiments the wave breaking type, breaking criteria and frequency selectivity of energy dissipation due to breaking depend strongly on amplitude-phase frequency structure of waves and cannot be predicted correctly on the base input wave parameters (height and period) and average bottom slope of coastal zone. To predict correctly the breaking type and position of breaking it is need to analyze the scenario of nonlinear wave transformation in the coastal zone.

The discovered phase-frequency structure of breaking waves permit to explains the empirical fact that Plunging breaking waves wash out the beaches, then Spilling breaking waves restore the beaches according Bailard 's formula for sediment transport and taking in account the undertow. Under the plunging, the wave component of sediment transport equal to zero and undertow wash out the sediment to the sea. Under spilling wave the wave component of sediment transport is high, directed to shore and bigger then undertow component directed to sea so the sediment stored at the shoreline.

\section{ACKNOWLEDGMENTS}

This work was supported by the Russian Science Foundation (the project 14-50-00095) and partly by the Russian Foundation for Basic Researches (the project 18-55-45026).

\section{REFERENCES}

Battjes J.A. 1974. Surf similarity, Proc. 14th Conference Coastal Engineering. New York, ASCE, 466480.

Battjes J.A. and J.P.F.M. Janssen. 1978. Energy loss and set-up due to breaking of random waves, Proceedings of $14^{\text {th }}$ International Conference on Coastal Engineering, ASCE, 466-480.

Eldeberky Y., J.A. Battjes. 1996. Spectral modeling of wave breaking: application to Boussinesq equations. J. Geoph. Res., 101, C1, 1253-1264. 
Kuznetsov S.Yu., Ya.V. Saprykina. 2004. Frequency-dependent energy dissipation of irregular breaking waves, Water Resources, 31, 4, 384-392.

Madsen, P.A. and O.R. Sørensen. 1993. Bound Waves and Triad Interactions in Shallow Water, Journal of Ocean Engineering, 20, 4, 359-388.

Mase H., J. Kirby. 1992. Hybryd freguency-domain KdV eguation for random wave transformation, Proc.23d Int.Conf.Coast.Engrng., Venice., 474-487.

Saprykina Y., S. Kuznetsov, B. Divinskii. 2017. Influence of processes of nonlinear transformations of waves in the coastal zone on the height of breaking waves, Oceanology, 57, 3, 383-393.

Saprykina Y.V., S.Y.Kuznetsov, M.N.Shtremel, N.K. Andreeva. 2013. Scenarios of nonlinear wave transformation in the coastal zone, Oceanology, 53, 4, 422-431.

Thornton E.T., R.T. Guza. 1983 . Transformation of wave height distribution, J. Geoph. Res., 88, 59255938. 